\title{
Pathologic Classification of Rhabdomyosarcomas and Correlations with Molecular Studies
}

\author{
David M. Parham, M.D. \\ Department of Pathology, Arkansas Children's Hospital and University of Arkansas for Medical Sciences, \\ Little Rock, Arkansas
}

\begin{abstract}
Rhabdomyosarcoma, the most common soft-tissue malignancy of childhood and adolescence, comprises a group of differing pathobiologic entities linked by their common propensity for formation of neoplastic skeletal muscle, a feature that results from biological forces related to aberrant transcription signals and the resultant production of myogenic proteins. At a molecular level, however, rhabdomyosarcomas form a heterogeneous group that can be subdivided into distinct clinicopathologic entities based on morphologic appearance and genetic makeup. These differing morphologic features were recognized in the mid-1900s by Horn and Enterline with their division of rhabdomyosarcomas into embryonal, alveolar, botryoid, and pleomorphic subtypes. More recent histologic and biologic studies have resulted in description of additional entities, such as spindle cell and anaplastic rhabdomyosarcoma, and refinements in recognition of the original entities, such as solid-alveolar rhabdomyosarcoma. Familiarity with newer classifications and their relationship to molecular aberrations is key to stratifying patients on current therapeutic protocols and proposed innovative genetic therapies.
\end{abstract}

KEY WORDS: Classification, Cytogenetics, Molecular biology, Pathology, Review, Rhabdomyosarcoma.

Mod Pathol 2001;14(5):506-514

Although relatively rare in adults, rhabdomyosarcomas are the most common soft-tissue malignancy in children and adolescents, in whom they comprise approximately $60 \%$ of sarcomas reported per annum (1). In many ways, the histology of these neoplasms is analogous to myogenesis in the de-

Copyright (C) 2001 by The United States and Canadian Academy of Pathology, Inc.

VOL. 14, NO. 5, P. 506, 2001 Printed in the U.S.A.

Date of acceptance: January 22, 2001.

Presented at the Pathology Committee Symposium, Children's Oncology Group, Tampa, Florida, April 14, 2000.

Address reprint requests to: David M. Parham, M.D., Slot 820, Arkansas Children's Hospital, 800 Marshall Street, Little Rock, AR 72202; e-mail: parhamdavidm@exchange.uams.edu; fax: 501-320-3912. veloping embryo, yielding clues to the biology of these lesions. For this reason, it is more correct to define rhabdomyosarcoma as a tumor derived from primitive mesenchyme and exhibiting a profound tendency towards myogenesis than to define it as a cancer arising from skeletal muscle. The former concept was eloquently expressed by Masson (2), who referred to rhabdomyosarcomas as "rhabdopoietic sarcomas," and it better explains the observation that more tumors arise from viscera and axial soft tissues than from the extremities (3). Historically, this is a well-established phenomenon, considering Stafford's report in 1839 (4) of a probable prostatic rhabdomyosarcoma in a 5-year-old child, and the first series of these tumors, reported in 1894 (5), in which the majority of cases involved the urogenital tract.

\section{Standard Classification}

Ironically, it was not the striking histologic differentiation of rhabdomyosarcomas that led to Guersant's (6) description of the first reported subcategory of these neoplasms but the gross appearance of a tumor arising from the vagina of a young girl. This polypoid lesion resembled a bunch of grapes, leading to its designation as a sarcoma botryoides, from the Greek term botryos. Botryoid rhabdomyosarcoma, the current name for sarcoma botryoides, now comprises a clinically and pathologically distinctive form of rhabdomyosarcomas. These lesions should abut an epithelial surface, such as that of the bladder, bile duct, vagina, or conjunctiva, and project into the lumen as multinodular excrescences of variable size. Intriguingly, a similar phenomenon occurs in rhabdomyomatous Wilms tumors that involve the renal pelvis $(7,8)$. The microscopic sine qua non of botryoid rhabdomyosarcoma, prerequisite for the diagnosis by the current classification (9), is the cambium layer (Fig. 1). This subepithelial condensation of tumor cells derives its name from the from the cambium layer of plants, a compressed subsurface zone of cells in stems and roots that gives rise to phloem and xy- 


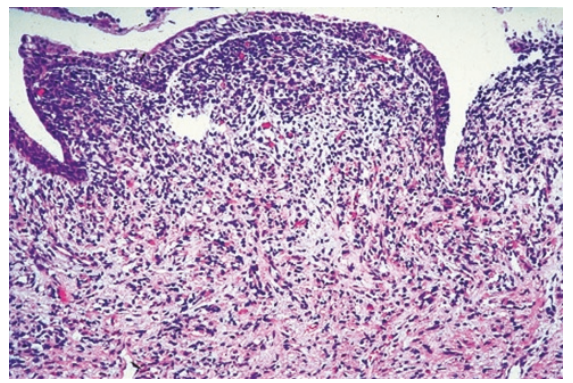

FIGURE 1. Botryoid rhabdomyosarcoma. A small-cell neoplasm abuts an epithelial surface, with condensation of tumor cells in the immediate subepithelial zone. Reprinted from Parham DM, editor: Pediatric Neoplasia: Morphology and Biology. Philadelphia: LippincottRaven; 1996, by permission of Lippincott-Williams and Wilkins.

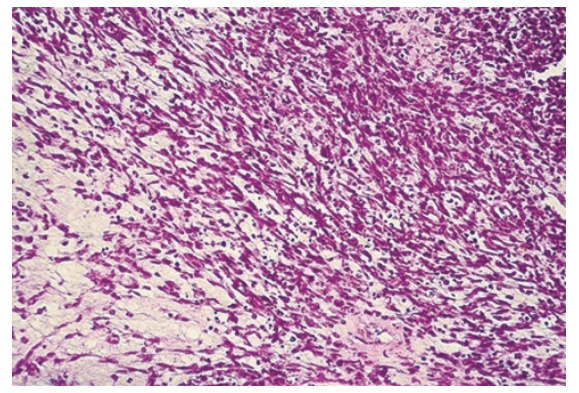

FIGURE 2. Embryonal rhabdomyosarcoma. This tumor contains dense condensations of rhabdomyoblasts amid foci with a loose myxoid stroma. Reprinted from Parham DM, editor: Pediatric Neoplasia: Morphology and Biology. Philadelphia: Lippincott-Raven; 1996, by permission of Lippincott-Williams and Wilkins.

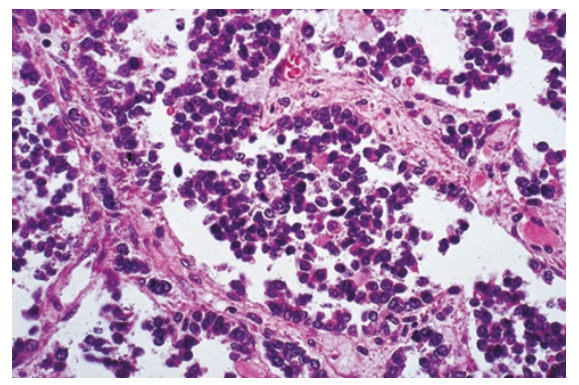

FIGURE 3. Alveolar rhabdomyosarcoma. Aggregates of primitive round cells appear to "float" within nests outlined by fibrous septa lined tumor cells in a picket row configuration. Reprinted from Parham DM, editor: Pediatric Neoplasia: Morphology and Biology. Philadelphia: Lippincott-Raven; 1996, by permission of Lippincott-Williams and Wilkins.

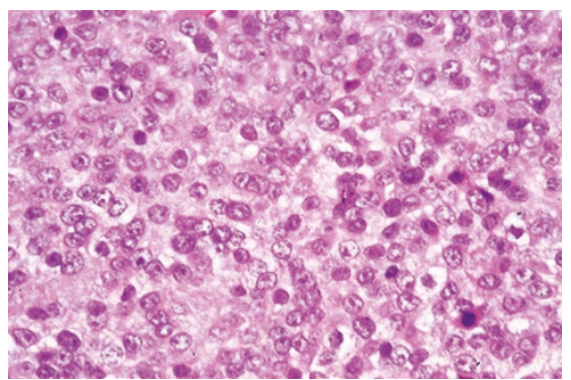

FIGURE 4. Alveolar rhabdomyosarcoma, solid variant (monomorphous round cell tumor of Palmer). The neoplasm is composed of a densely cellular sheet of primitive rhabdomyoblasts with monomorphous, round nuclei and no discernible septa. This tumor is also illustrative of the monomorphous round cell category of the Palmer classification. Reprinted from Parham DM, editor: Pediatric Neoplasia: Morphology and Biology. Philadelphia: Lippincott-Raven; 1996, by permission of Lippincott-Williams and Wilkins.

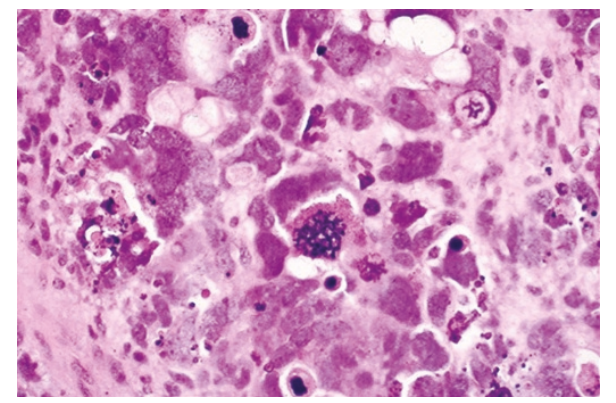

FIGURE 5. Anaplastic rhabdomyosarcoma, diffuse. Anaplastic rhabdomyosarcomas are characterized by cells with enlarged, pleomorphic, hyperchromatic nuclei. In this example, they are arrayed in a discrete cluster, indicative of the clonal proliferation that defines diffuse anaplasia. Note the multipolar mitosis, also frequently seen in these neoplasms. Reprinted from Parham DM, editor: Pediatric Neoplasia: Morphology and Biology. Philadelphia: Lippincott-Raven; 1996, by permission of Lippincott-Williams and Wilkins.

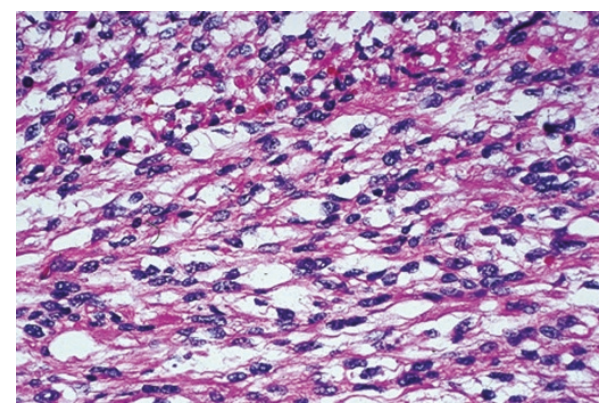

FIGURE 6. Spindle cell rhabdomyosarcoma (type A lesion). This neoplasm contains relatively differentiated spindle cells having cytologic features reminiscent of smooth muscle tumors. In the Palmer classification, spindle cell rhabdomyosarcomas were known as type A lesions. Reprinted from Parham DM, editor: Pediatric Neoplasia: Morphology and Biology. Philadelphia: Lippincott-Raven; 1996, by permission of Lippincott-Williams and Wilkins.

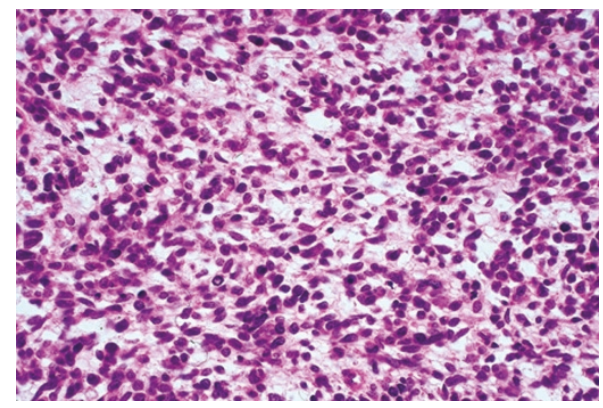

FIGURE 7. Embryonal sarcoma (International Society of Pediatric Oncology [SIOP] classification). In the SIOP classification, this tumor would qualify as an embryonal sarcoma because of its lack of discrete histologic evidence of myogenesis. However, with the International Classification, these tumors are included with embryonal rhabdomyosarcomas. Reprinted from Parham DM, editor: Pediatric Neoplasia: Morphology and Biology. Philadelphia: Lippincott-Raven; 1996, by permission of Lippincott-Williams and Wilkins.

lem. Whether botryoid rhabdomyosarcomas are a biologically distinct entity or a site-specific variant of embryonal rhabdomyosarcomas remains a subject of debate, and biologic studies to date have not yielded a molecular distinction.

Microscopic observations led to description of embryonal rhabdomyosarcoma, the next variant of 
rhabdomyosarcoma to be discovered. This was so named in 1894 by Berard (10), who termed it a tumeur embryonnaire du muscle striae. The histologic features of this tumor, with its zones of loose and dense cellularity, remarkably recapitulate normal embryonal myogenesis, in which loose primitive mesenchyme condenses to form nascent muscle (Fig. 2). The tumor cells composing embryonal rhabdomyosarcomas variably exhibit all cellular phases of myogenesis, from stellate undifferentiated mesenchymal cells to elongated myoblasts, multinucleated myotubes, and fully differentiated myofibers. The latter phases become more prevalent after combination chemotherapy and irradiation, which induces terminal differentiation (11). As a general rule, embryonal rhabdomyosarcomas arise from the genitourinary tract, head and neck, and abdomen and affect infants and young children. Thus, both histologically and clinically, they share features with other embryonal neoplasms of childhood, such as Wilms tumors, hepatoblastomas, pancreatoblastomas, and neuroblastomas. They similarly occur as part of the neoplastic spectrum of the Beckwith-Wiedemann syndrome, a familial lesion with biologic implications for epigenetic inheritance and imprinting $(12,13)$.

In 1946, Stout, one of the premier soft-tissue pathologists of the twentieth century, made the initial description of the next published variant of rhabdomyosarcoma, the pleomorphic subtype (14). Pleomorphic rhabdomyosarcomas arise almost exclusively in the soft tissues of adults and comprise part of the spectrum of lesions known as "malignant fibrous histiocytoma" (15). They form spindle cell lesions with a whorled or storiform pattern and containing cells with enlarged, hyperchromatic nuclei. Because the tumor cells only rarely display frank myogenesis, there has been controversy as to the very existence of this neoplasm, but immunohistochemical studies have confirmed their myogenetic potential and verified their nosologic status (16). In the rare pleomorphic rhabdomyosarcoma seen in the pediatric age group, there is usually another component (17), so that this category currently is not used for pediatric subtyping and has been subsumed into the anaplastic and spindle cell subtypes (see later discussion).

In 1956 (18), Riopelle and Theriault described the final category of traditionally recognized rhabdomyosarcomas, alveolar rhabdomyosarcoma. Using only six cases of their own and comparing them with other published tumors, they engendered a new species of small-cell tumor that was distinctive from similar lesions tending to arise in adolescents, such as lymphoma and Ewing sarcoma. In those halcyon days preceding the advent of immunohistochemistry, pattern recognition played a solo role in pathologic diagnosis, but it lacked sufficient def- inition to deal with histologically indistinguishable, undifferentiated, small round cell tumors. Thus, Riopelle and Theriault focused on a key defining pattern, the alveolar structure formed by the fibrous septa of these neoplasms, which offered a practical, reproducible means of diagnosis (Fig. 3). These fibrous septa are composed of collagenous fibrovascular tissue that forms a scaffolding suspending attached rhabdomyoblasts and circumscribing central clusters of tumor cells that appear to float in alveolar spaces. Of note is that in these neoplasms, Riopelle and Theriault described solid areas lacking fibrosis and resembling lymphoma, a phenomenon further noted by Enzinger and Shiraki (19) in one half of the AFIP series and more extensively used by recent classifications that recognize a solid pattern in so-called alveolar tumors.

Drawing from the key reports described above and using their own series of 39 cases, in 1958, Horn and Enterline (20) derived the first commonly used subclassification system for rhabdomyosarcomas (Table 1). This system came into common use in the latter part of the 1900s and appeared to work well from a clinicopathologic standpoint, as it predictably defined tumor types likely to occur in given age ranges and anatomic sites. Using univariate analysis of Intergroup Rhabdomyosarcoma Study (IRS) material, Newton et al. (17) demonstrated the potential prognostic value of standard rhabdomyosarcoma classification. However, even luminaries such as Stout and Lattes (21) felt that subclassification had little bearing on survival, and in one report (22), Gonzalez-Crussi and Black-Schaffer lamented that it was based on poorly defined criteria, resulted in marked disparities in studies, and disregarded morphologic heterogeneity. Thus, the stage was set for further refinements in rhabdomyosarcoma classification.

\section{Cytohistological (Palmer) Classification}

The cytohistological classification promulgated by Palmer et al. began as a retrospective investigation of IRS-I and -II material maintained at the IRS Pathology Center. Beckwith and Palmer (23) had published a landmark histopathologic study that described the prognostic features of pediatric renal tumors, and Palmer applied similar methods to the study of rhabdomyosarcoma. In this manner, Palmer used no preconceptions about rhabdomyosarcoma histology, but rather he catalogued a series of recurring cytologic appearances and tested their value using statistical analysis. His resultant classification is listed in Table 1. Palmer found his classification to be more predictive than the Horn and Enterline classifications performed by the IRS pathologists, and he and Foulkes estimated that the 
TABLE 1. Classifications of Rhabdomyosarcomas, with Comparisons among Schemae

\begin{tabular}{|c|c|c|c|c|}
\hline Horn-Enterline (20) & Palmer $(24)^{\mathrm{a}}$ & SIOP (27) & NCI (29) & International (9) \\
\hline Embryonal & $\begin{array}{l}\text { "Mixed" } \\
\text { Type A }\end{array}$ & $\begin{array}{l}\text { Embryonal } \\
\text { Dense, well differentiated } \\
\text { Dense, poorly differentiated } \\
\text { Not otherwise specified } \\
\text { Loose, nonbotryoid }\end{array}$ & $\begin{array}{l}\text { Embryonal } \\
\text { Leiomyomatous }\end{array}$ & $\begin{array}{l}\text { Embryonal } \\
\text { Spindle cell }\end{array}$ \\
\hline Botryoid & & $\begin{array}{l}\text { Loose, botryoid } \\
\text { Embryonal sarcoma }\end{array}$ & Botryoid & Botryoid \\
\hline & Anaplastic & \multicolumn{3}{|c|}{ Pleomorphic embryonal } \\
\hline Alveolar & \multicolumn{2}{|c|}{ Monomorphous round cell Alveolar } & $\begin{array}{l}\text { Alveolar } \\
\text { Solid variant }\end{array}$ & Alveolar (includes solid variant) \\
\hline Pleomorphic & Anaplastic & Pleomorphic & Pleomorphic & $\begin{array}{l}\text { Rhabdomyosarcoma, not otherwise specified } \\
\text { Sarcoma, not otherwise specified } \\
\text { Undifferentiated sarcoma }\end{array}$ \\
\hline
\end{tabular}

SIOP, International Society for Pediatric Oncology; NCI, National Cancer Institute.

a Mixed tumors in the Palmer classification might equally apply to alveolar and embryonal rhabdomyosarcomas, as his category is independent of histology; most, however, are embryonal. Anaplasia might also be seen in both histologies.

presence of unfavorable histology increased the risk of death by a factor of 3.77 (24).

Palmer's cytohistologic classification was based on nuclear morphology rather than cytoplasmic differentiation. As such, his work foreshadowed observations made in subsequent newer classifications that refined the standard Horn-Enterline system. Among these findings were that tumors composed exclusively of small round cells (monomorphous round cell rhabdomyosarcoma; Fig. 4) were bad actors, that neoplasms with large anaplastic cells (anaplastic rhabdomyosarcoma; Fig. 5) connoted a bad prognosis, and that leiomyosarcomalike lesions (Type A rhabdomyosarcomas; Fig. 6) were relatively low grade. Unfortunately, Palmer et al.'s work was never published in a refereed journal, only as meeting abstracts (24-26).

\section{SIOP Classification}

Using methods similar to those of Palmer, in 1989, Caillaud et al. (27) published a classification used to test a pediatric sarcoma registry from a large multi-institutional study, that of the International Society of Pediatric Oncology (SIOP). The IRS and SIOP studies diverged, however, in that the SIOP group concentrated on cytologic differentiation and cellular density rather than nuclear features (see Table 1). In this manner, embryonal rhabdomyosarcomas were subdivided into loose or dense, botryoid or non-botryoid, poorly differentiated or well differentiated, or not otherwise specified (NOS). Alveolar and pleomorphic tumors retained the conventional definitions of Horn and Enterline, and a new category, embryonal sarcoma (Fig. 7), arose; these sarcomas were characterized by their low-power similarity to embryonal rhabdomyosarcomas but high-power lack of obvious myogenesis by routine staining (28). Redefinition of embryonal sarcomas becomes necessary when one adds muscle immunostains to the diagnostic armamentarium, as they are usually positive in these tumors, and chemotherapy also induces the tumor cells to exhibit myogenic differentiation (28). As a result, embryonal sarcomas are considered a primitive form of embryonal rhabdomyosarcoma in our current classification (9).

In the initial SIOP review, superior outcomes were seen in three subtypes of rhabdomyosarcoma: loose botryoid, embryonal NOS, and dense welldifferentiated embryonal. In general, welldifferentiated tumors did better than alveolar and poorly differentiated ones. To put these results into perspective with those of the other classifications, it should be noted that SIOP alveolar tumors were of the standard variety with fibrous septa but that solid alveolar tumors had been included with embryonal lesions as per the Horn-Enterline classification. One may thus assume that a proportion of the poorly differentiated embryonal rhabdomyosarcomas in the SIOP classification might correspond to solid alveolar rhabdomyosarcomas of the National Cancer Institute (NCI) classification, as described below. Of interest is that the primitive embryonal sarcomas fared better than standard embryonal rhabdomyosarcomas in a subsequent SIOP review (28).

\section{National Cancer Institute (NCI) Classification}

The recognition of solid alveolar rhabdomyosarcoma represented the major revision made by the NCI classification (Table 1), published in 1990 by Tsokos et al. (29). As stated above, in many ways, the inclusion of solid alveolar tumors lacking fibrous septa into the alveolar category was a reinvention of the original intent (Fig. 4). Riopelle and Theriault (18) had noted that these foci occurred 
often at the periphery of the lesions, and Enzinger and Shiraki's AFIP series (19) had contained these areas in one half of alveolar tumors. The key to recognition of alveolar tumors was the round, monomorphous, lymphoma-like, nuclear cytology, as had been presciently noted by Palmer and Foulkes (24). In a retrospective study of the NCI classification using a large cohort of rhabdomyosarcomas treated at the NCI and St. Jude Children's Research Hospital, the clinical outcome of solid tumors paralleled that of typical alveolar rhabdomyosarcomas. In addition, NCI classification was an independent prognostic factor in multivariate analysis, whereas conventional Horn-Enterline histology was predictive of outcome only in univariate statistical study.

Subcategories of embryonal histology also constituted part of the NCI classification; these included pleomorphic embryonal, leiomyomatous embryonal, and embryonal with aggressive foci (29). By statistical analysis, these subclassifications of embryonal rhabdomyosarcoma did not appear to affect clinical outcome in the combined NCI/St. Jude review.

Categorization of rhabdomyosarcomas was tremendously aided by a major discovery made in the early 1980s, namely, that alveolar rhabdomyosarcoma is cytogenetically characterized by a reciprocal translocation, the $\mathrm{t}(2 ; 13)(\mathrm{q} 35 ; \mathrm{q} 14)$. Using this karotypic aberration, which appeared unique to alveolar tumors, it was possible to prove that solidalveolar rhabdomyosarcomas possess biologic as well as clinicopathologic identity with typical alveolar tumors (30).

\section{International Classification}

As this plethora of subcategorization arose, it became evident that rhabdomyosarcoma classification was heading down the same muddy road of confusion that had been plaguing lymphoma classification. Thus, using the model developed by the Working Classification Group for Non-Hodgkin's Lymphoma (31), Newton and colleagues at the IRS undertook a similarly promethean task, that of testing each of the systems described above and authoring a new international classification. The ultimate goal of this group was to stratify rhabdomyosarcoma into prognostically significant and diagnostically consistent morphologic subgroups. To this end, Newton and colleagues assembled a panel of experts representing each classification, tested the overall agreement reached among these pathologists when using all schemas, and devised a new system based on the level of agreement and power of prognostic prediction $(9,32)$. The result, an International Classification for Rhabdomyosarcoma, is shown in Table 1. The diagnostic categories of rhabdomyosarcoma, not otherwise specified, and sarcoma, not otherwise specified, were respectively retained for cases in which rhabdomyosarcoma could be diagnosed but not subtyped or in which the diagnosis was strongly suspected but not verifiable because of suboptimal biopsy material (9). The IRS Pathologic Review Committee (now part of the Children's Oncology Group) has continued to use this system to the present time (33).

The basic rule for application of the International Classification is that the key diagnostic features used for embryonal and alveolar variants of rhabdomyosarcoma, as described above, have been retained. The category of alveolar rhabdomyosarcomas been expanded to include solid variants (see earlier in this article) that lack fibrous septa (Fig. 4). Botryoid rhabdomyosarcoma has also been retained as a diagnostic category, with features as described above, with the caveat that a cambium layer (Fig. 1) is essential for diagnosis. Finally, a new category, spindle cell rhabdomyosarcoma, has been added, with features as described below. Because of pleomorphic rhabdomyosarcoma's rarity in children and its overlap with anaplastic rhabdomyosarcoma (see below), it is not included in the International Classification.

One feature of particular note is that some rhabdomyosarcomas contain histologic features of multiple subtypes. In current classification parlance, the presence of any alveolar element translates into a bad prognosis (34). The biologic basis for these mixed tumors is currently unknown, although some studies suggest that even the embryonal elements of "bad" tumors have genetic features of alveolar rhabdomyosarcoma $(35,36)$. Interestingly, alveolar rhabdomyosarcomas frequently show amplification of MYCN, a phenomenon observed only in the alveolar component of mixed tumors (37).

\section{Newer Entities}

Inclusion of one new diagnostic subcategory of rhabdomyosarcoma, spindle cell rhabdomyosarcoma, was deemed necessary when the International Classification was devised. Taking their cue from the older studies of Palmer of "type A" superior prognosis tumors $(25,26)$, Cavazzana and colleagues (38) in 1992 published a retrospective analysis of these lesions, which they renamed "spindle cell rhabdomyosarcomas." Using their own clinical data and morphologic studies, they confirmed the excellent clinical outcome of these lesions and carefully described their histologic features. Spindle cell rhabdomyosarcomas primarily arose in paratesticular locations and exhibited a whorled spindly appearance akin to that of smooth muscle tumors (Fig. 6). Additional retrospective analyses were then 
performed by IRS pathologists, who reconfirmed the uniqueness of these tumors (39). They thus were included as a separate category in the International Classification (9). One histologic feature noted in Cavazzana's study (38) was strong immunopositivity for titin, a marker of terminal differentiation.

Another new entity that was considered for inclusion in the International Classification but ultimately not included was the anaplastic rhabdomyosarcoma. Anaplastic tumors had constituted a poor prognosis group of the Cytohistologic Classification of Palmer, but their definition was somewhat poorly defined because it was based solely on the presence of a single atypical multipolar mitosis. A reanalysis of the slides and clinical data published in 1993 by the IRS Pathology Center (40) indicated that enlarged, hyperchromatic nuclei akin to those in anaplastic Wilms tumor (23) were also key to the diagnosis. However, the critical factor in determining the poor clinical outcome of these tumors appeared to be whether the anaplastic cells were aggregated in a clone-like cluster or sprinkled in a haphazard, single-cell fashion; the former feature appeared to be a bad-prognosis factor. Preliminary data from immunohistochemical studies indicates that the anaplastic cells contain abnormal p53 (41). Work on additional tumors is ongoing at the IRSG Pathology Center, and prospective data is accumulating to determine whether these tumors will retain their bad connotation and deserve special therapy. At present, they are not included in the International Classification (Table 1).

For the practicing pathologist, anaplasia is best recognized on low-power examination as areas of cellular and nuclear enlargement. Focusing on these areas should reveal hyperchromasia and pleomorphism in affected cells (Fig. 5). Although currently not a critical feature, the presence of multipolar mitoses is frequently noted. The significance of these findings lies in the fact that a limited number of reports has associated these cells with poor outcome, particularly if discrete, clonal populations are seen. However, recognition of anaplasia does not affect clinical management at present on IRSG protocols, pending accumulation of additional prospective data.

\section{Biologic Studies Related to Rhabdomyosarcoma Classification}

Work continued apace on characterization of the $t(2 ; 13)$ translocation of alveolar rhabdomyosarcoma, with the discovery that the genetic breakpoints resulted in a fusion of the genes $P A X 3$ and FRHR (formerly $A L V$ ). The resultant chimeric protein causes aberrant transcription resulting in both unrestrained cellular division and initiation of a myogenesis program $(35,42)$. Using this data, in 1995, Downing and colleagues (43) developed a molecular diagnostic test to detect this aberration using reverse transcriptase-polymerase chain reaction (RT-PCR).

Another discovery was that an another member of the PAX family, PAX7, fused with FRHR in an alternate translocation present in some alveolar rhabdomyosarcoma, the $\mathrm{t}(1 ; 13)$ (p36; 14$)$. The importance of this discovery has recently been heightened by indications that tumors with this translocation behave in a manner more akin to that of embryonal tumors than alveolar ones (44), a phenomenon perhaps related to differing mechanisms of transcription between the PAX 7/FRHR and PAX3/ FRHR fusions. A surprisingly large subset of alveolar rhabdomyosarcomas have appeared to show no evidence of a translocation $(43,45)$; investigation of the molecular nature of these fusion-negative alveolar rhabdomyosarcomas is in progress.

Unlike the case in alveolar tumors, efforts to discover a single, key genetic mutation in embryonal rhabdomyosarcomas have been fruitless. It is apparent that many of these tumors exhibit loss of heterozygosity (LOH) at chromosome $11 \mathrm{p}$, and this discovery by Scrable et al. (46) was used to proposed a classification based on molecular features rather than histologic ones. $\mathrm{LOH}$ in this region links embryonal rhabdomyosarcomas with other embryonal neoplasms, such as Wilms tumors and hepatoblastomas (47), and constitutional lesions such as the Beckwith-Wiedemann syndrome (48). A perhaps-related phenomenon is loss of genetic imprinting in genes at this locus $(49,50)$. Although a single genetic lesion cannot be identified, aberrations affecting the methylation status of key genes such as MyoD (51) and p21 (52) have been identified. These findings suggest that the causation of embryonal rhabdomyosarcoma may be epigenetic rather than genetic, which would explain the association of rhabdomyosarcoma with alterations of imprinting. This hypothesis might also explain the frequent hyperdiploidy $(53,54)$ and chromosomal duplications $(55,56)$ seen in embryonal rhabdomyosarcomas, as lesions affecting methylation genes can lead to these phenomena (57-59).

\section{Modern-Day Diagnosis of Rhabdomyosarcoma}

Like classification, modern-day diagnosis of rhabdomyosarcoma is often a difficult proposition. However, some of the tools we have learned to use in classifying rhabdomyosarcoma are equally effective for ancillary diagnosis. In the 21st century, immunohistochemistry has attained ascendancy as the foremost method of diagnostic confirmation. A variety of muscle markers have been identified; a partial listing is seen in Table 2. Myoglobin, one of 
TABLE 2. Immunohistochemical Stains Useful for Rhabdomyosarcoma Diagnosis

\begin{tabular}{ll}
\hline \multicolumn{1}{c}{ Type } & \multicolumn{1}{c}{ Marker } \\
\hline Cytoplasmic nonfilamentous & Myoglobin \\
& Creatine kinase M \\
Cytoplasmic, filamentous & Desmin \\
& Muscle-specific actin \\
Nuclear & Sarcomeric myosin \\
& MyoD \\
& Myogenin \\
\hline
\end{tabular}

the first markers identified, suffers from its relative lack of sensitivity, as only differentiated cells express it. Desmin and muscle-specific actin have been used most extensively, gaining much popularity during the late 1980s. These filamentous proteins are relatively sensitive, but they are also expressed by a variety of cells other than rhabdomyoblasts, including smooth muscle, myofibroblasts, pericytes, and myoepithelium. Among sarcomas, peripheral neuroectodermal tumors (PNET), myofibrosarcomas, leiomyosarcomas, malignant peripheral nerve sheath tumors, and desmoplastic small-cell tumors may express desmin and/or muscle actin. Perhaps the most sensitive and specific group of proteins useful in immunohistochemical diagnosis of rhabdomyosarcoma are the muscle transcription factors MyoD and myogenin. These proteins, which define the earliest events in molecular determination of myoblastic lineage, are paradoxically expressed at high levels in most rhabdomyosarcomas, even though the cells often show little differentiation. Immunohistochemical techniques using heat retrieval allows use of these markers in paraffin sections, although this process tends to decrease staining and to introduce an element of nonspecific cytoplasmic positivity. Thus, only nuclear staining should be considered positive. The pattern of staining appears to predict classification, as a heterogeneous pattern is more typical of embryonal rhabdomyosarcoma, whereas strong, diffuse staining is a feature of alveolar rhabdomyosarcoma (60).

Other diagnostic approaches are appropriate with some tumors, as the differential diagnosis of rhabdomyosarcoma comprises the entire breadth of small, round cell tumors, including neuroblastoma, the Ewing family of tumors (Ewing sarcoma and PNET), lymphoma, and desmoplastic small cell tumor. Spindle cells also are common in rhabdomyosarcomas, prompting consideration of fibrosarcoma and myofibrosarcoma, leiomyosarcoma, malignant fibrous histiocytoma and its congeners, malignant peripheral nerve sheath tumor (particularly Triton tumor), epithelioid sarcoma, and synovial sarcoma. Occasional rhabdomyosarcomas display a bewildering phenotype; even monoclonal light-chain and B-cell marker positivity are possible
(61). Ectomesenchymomas are defined by their mixture of muscle and neural elements (62). Some aggressive rhabdomyosarcomas present with no defined primary, extensive bone marrow involvement, and even leukemic spread (63). Thus, it is sometimes desirable to resort to molecular testing with fusion markers or cytogenetics. In circumstances in which histologic features do not match immunohistochemical stains, I find it particularly helpful to use genetic studies and electron microscopy.

Another problematic feature of rhabdomyosarcomas arises with excisions or biopsies performed after multimodality therapy. Treatment induces not only necrosis and shrinkage but also fibrosis and cytodifferentiation (64). The terminal differentiation so induced can be so striking that the neoplastic myoblasts recapitulate normal muscle, particularly entrapped, regenerative myofibers. In tumors involving skeletal muscle, an unresolvable Gordian knot may arise that cannot be completely solved with current techniques. At present, the clinical significance of terminally differentiated tumor is unclear, although some limited, retrospective studies would indicate that it is nontumorigenic (65). The exquisite specificity of myoglobin in terminally differentiated myocytes makes it a useful marker of differentiated tumor in non-skeletal musclecontaining tissues like urinary bladder or prostate. MyoD and myogenin are less helpful because their expression is usually down-regulated with terminal differentiation.

\section{SUMMARY}

It is apparent that rhabdomyosarcoma comprises a group of morphologically similar but biologically diverse lesions. The latest classification systems represent attempts to synthesize clinically relevant subgroups based on histologic appearance and verified by molecular genetic techniques. Using these tools, rhabdomyosarcomas can be subdivided into superior, good, and bad prognosis groups. Work with some categories, such as anaplastic rhabdomyosarcomas, is ongoing. Evolution of these ideas will doubtlessly progress as biologic and clinicopathologic studies yield additional data.

\section{REFERENCES}

1. Miller RW, Young JL Jr, Novakovic B. Childhood cancer. Cancer 1995(1 Suppl);75:395-405.

2. Masson P. Human tumors. Histology, diagnosis, and technique. 2nd ed. Detroit, MI: Wayne State University Press; 1970.

3. Donaldson SS. Rhabdomyosarcoma. Contemporary status and future directions: The Lucy Wortham James Clinical Research Award. Arch Surg 1989;124:1015-20. 
4. Stafford RA. A case of enlargement from melanoid tumor of the prostate gland in a child five years of age. Med Chir Trans 1839;22:218.

5. Wolfensberger R. Uber ein rhabdomyom der speiserohre. Beitr Pathol Anat Allg Pathol 1894;15:491.

6. Guersant MP. Polypes du vagin chez une petite fille de treize mois. Monit Hopit 1854;2:187.

7. Mahoney JP, Saffos RO. Fetal rhabdomyomatous nephroblastoma with a renal pelvic mass simulating sarcoma botryoides. Am J Surg Pathol 1981;5:297-306.

8. Parham DM. Pediatric neoplasia: morphology and biology. Philadelphia: Lippincott-Raven; 1996.

9. Newton WA Jr, Gehan EA, Webber BL, Marsden HB, van Unnik AJM, Hamoudi AB, et al. Classification of rhabdomyosarcoma and related sarcomas: pathologic aspects and proposal for a new classification-an Intergroup Rhabdomyosarcoma Study. Cancer 1995;76:1073-85.

10. Berard M. Tumeur embryonnaire du muscle striae. Lyon Med 1894;77:52.

11. Parham DM. The molecular biology of childhood rhabdomyosarcoma. Semin Diag Pathol 1994;11:39-46.

12. Reid LH, Davies C, Cooper PR, Crider-Miller SJ, Sait SN, Nowak NJ, et al. A 1-Mb physical map and PAC contig of the imprinted domain in 11p15.5 that contains TAPAl and the BWSCR1/WT2 region. Genomics 1997;43:366-75.

13. Besnard-Guerin C, Newsham I, Winqvist R, Cavenee WK. A common region of loss of heterozygosity in Wilms' tumor and embryonal rhabdomyosarcoma distal to the D11S988 locus on chromosome 11p15.5. Hum Genet 1996;97:163-70.

14. Stout AP. Rhabdomyosarcoma of the skeletal muscles. Ann Surg 1946;123:447-72.

15. Fletcher CDM. Pleomorphic malignant fibrous histiocytoma: Fact or fiction? A critical reappraisal based on 159 tumors diagnosed as pleomorphic sarcoma. Am J Surg Pathol 1992; 16:213-28.

16. Wesche WA, Fletcher CDM, Dias P, Houghton PJ, Parham DM. Immunohistochemistry of MyoD1 in adult pleomorphic soft tissue sarcomas. Am J Surg Pathol 1995;19:261-9.

17. Newton WA Jr, Soule EH, Hamoudi AB, Reiman HM, Shimada H, Beltangady M, et al. Histopathology of childhood sarcomas, Intergroup Rhabdomyosarcoma Studies I and II: clinicopathologic correlation. J Clin Oncol 1988;6:67-75.

18. Riopelle JL, Thériault JP. Sur une forme méconnue de sarcome des parties molles: le rhabdomyosarcome alvéolaire. Ann Anat Pathol 1956;1:88-111.

19. Enzinger FM, Shiraki M. Alveolar rhabdomyosarcoma: an analysis of 110 cases. Cancer 1969;24:18-31.

20. Horn RC, Enterline HT. Rhabdomyosarcoma: a clinicopathologic study of 39 cases. Cancer 1958;11:181-99.

21. Stout AP, Lattes R. Atlas of tumor pathology. 2nd series, Fascicle I: tumors of the soft tissues. Washington, DC: Armed Forces Institute of Pathology; 1967.

22. Gonzalez-Crussi F, Black-Schaffer S. Rhabdomyosarcoma of infancy and childhood: problems of morphologic classification. Am J Surg Pathol 1979;3:157-71.

23. Beckwith JB, Palmer NF. Histopathology and prognosis of Wilms tumors: results from the First National Wilms' Tumor Study. Cancer 1978;41:1937-48.

24. Palmer NF, Foulkes M. Histopathology and prognosis in the second Intergroup Rhabdomyosarcoma Study (IRS II) [Abstract]. Proc ASCO 1983;2:229.

25. Palmer NF, Sachs N, Foulkes M. Histology and prognosis in rhabdomyosarcoma: a report of the Intergroup Rhabdomyosarcoma Study [Abstract]. Proc SIOP 1981;13:113.

26. Palmer NF, Sachs N, Foulkes M. Histology and prognosis in rhabdomyosarcoma (IRS-I) [Abstract]. Proc ASCO 1982;1: 170.

27. Caillaud JM, Gérard-Marchant R, Marsden HB, van Unnik AJM, Rodary C, Rey A, et al. Histopathologic classification of childhood rhabdomyosarcoma: a report from the International Society of Pediatric Oncology Pathology Panel. Med Pediatr Oncol 1989;17:391-400.

28. Wijnaendts LCD, van der Linden JC, van Unnik AJM, Delemarre JFM, Voute PA, Meijer CJLM. Histologic classification of childhood rhabdomyosarcomas: relationship with clinical parameters and prognosis. Hum Pathol 1994;25:900-7.

29. Tsokos M, Webber B, Parham D, Wesley R, Miser A, Miser JS, et al. Rhabdomyosarcoma: a new classification scheme related to prognosis. Arch Pathol Lab Med 1992;116:847-55.

30. Parham DM, Shapiro DN, Downing JR, Webber BL, Douglass EC. Solid alveolar rhabdomyosarcomas with the $t(2 ; 13)$ : report of two cases with diagnostic implications. Am J Surg Pathol 1994;18:474-8.

31. The Non-Hodgkin's Lymphoma Pathologic Classification Project. National Cancer Institute sponsored study of classifications of non-Hodgkin's lymphomas: summary and description of a working formulation for clinical usage. Cancer 1982;49:2112-35.

32. Asmar L, Gehan EA, Newton WA, Webber BL, Marsden HB, van Unnik AJM, et al. Agreement among and within groups of pathologists in the classification of rhabdomyosarcoma and related childhood sarcomas: report of an international study of four pathology classifications. Cancer 1994;74:257988.

33. Qualman SJ, Coffin CM, Newton WA, Hojo H, Triche TJ, Parham DM, et al. Intergroup Rhabdomyosarcoma Study. Update for pathologists. Pediatr Dev Pathol 1998;1:550-61.

34. Pappo AS, Shapiro DN, Crist WM, Maurer HM. Biology and therapy of pediatric rhabdomyosarcoma. J Clin Oncol 1995; 13:2123-39.

35. Tobar A, Avigad S, Zoldan M, Mor C, Goshen Y, Zaizov R. Clinical relevance of molecular diagnosis in childhood rhabdomyosarcoma. Diagn Mol Pathol 2000;9:9-13.

36. Anderson J, Renshaw J, McManus A, Carter R, Mitchell C, Adams S, et al. Amplification of the $\mathrm{t}(2 ; 13)$ and $\mathrm{t}(1 ; 13)$ translocations of alveolar rhabdomyosarcoma in small formalinfixed biopsies using a modified reverse transcriptase polymerase chain reaction. Am J Pathol 1997;150:477-82.

37. Hachitanda Y, Toyoshima S, Akazawa K, Tsuneyoshi M. $\mathrm{N}$-myc gene amplification in rhabdomyosarcoma detected by fluorescence in situ hybridization: its correlation with histologic features. Mod Pathol 1998;11:1222-7.

38. Cavazzana AO, Schmidt D, Ninfo V, Harms D, Tollot M, Carli M, et al. Spindle cell rhabdomyosarcoma: A prognostically favorable variant of rhabdomyosarcoma. Am J Surg Pathol 1992;16:229-35.

39. Leuschner I, Newton WA Jr, Schmidt D, Sachs N, Asmar L, Hamoudi A, et al. Spindle cell variants of embryonal rhabdomyosarcoma in the paratesticular region: A report of the Intergroup Rhabdomyosarcoma Study. Am J Surg Pathol 1993;17:221-30.

40. Kodet R, Newton WA Jr, Hamoudi AB, Asmar L, Jacobs DL, Maurer HM. Childhood rhabdomyosarcoma with anaplastic (pleomorphic) features: a report of the Intergroup Rhabdomyosarcoma Study. Am J Surg Pathol 1993;17:443-53.

41. Pascasio J, Triche T, Sorensen P, Barr F, Qualman S. p53 expression correlates with anaplasia in rhabdomyosarcoma (RMS) [Abstract]. Mod Pathol 2000;11:4P.

42. Khan J, Bittner ML, Saal LH, Teichmann U, Azorsa DO, Gooden GC, et al. cDNA microarrays detect activation of a myogenic transcription program by the PAX3-FKHR fusion oncogene. Proc Natl Acad Sci U S A 1999;96:13264-9.

43. Downing JR, Khandekar A, Shurtleff SA, Head DR, Parham DM, Webber BL, et al. Multiplex RT-PCR assay for the differential diagnosis of alveolar rhabdomyosarcoma and Ewing's sarcoma. Am J Pathol 1995;146:626-34. 
44. Kelly KM, Womer RB, Sorensen PH, Xiong QB, Barr FG. Common and variant gene fusions predict distinct clinical phenotypes in rhabdomyosarcoma. J Clin Oncol 1997;15: 1831-6.

45. Kullendorff CM, Donner M, Mertens F, Mandahl N. Chromosomal aberrations in a consecutive series of childhood rhabdomyosarcoma. Med Pediatr Oncol 1998;30:156-9.

46. Scrable H, Witte D, Shimada H, Seemayer T, Wang-Wuu S, Soukup S, et al. Molecular differential pathology in rhabdomyosarcoma. Genes Chromosomes Cancer 1989;1:23-35.

47. Koufos A, Hansen MF, Copeland NG, Jenkins NA, Lampkin $\mathrm{BC}$, Cavenee WK. Loss of heterozygosity in three embryonal tumours suggests a common pathogenetic mechanism. Nature 1985;316:330-4.

48. Sotelo-Avila C, Gooch WM. Neoplasms associated with the Beckwith-Wiedemann syndrome. Perspect Pediatr Pathol 1976;3:255-72.

49. Scrable H, Cavenee W, Ghavimi F, Lovell M, Morgan K, Sapienza C. A model for embryonal rhabdomyosarcoma tumorigenesis that involves genome imprinting. Proc Natl Acad Sci U S A 1989;86:7480-4.

50. Zhan S, Shapiro DN, Helman LJ. Activation of an imprinted allele of the insulin-like growth factor II gene implicated in rhabdomyosarcoma. J Clin Invest 1994;94:445-8.

51. Chen B, Dias P, Jenkins JJ, Savell VH, Parham DM. Methylation alterations of the MyoD1 upstream region are predictive of subclassification of human rhabdomyosarcomas. Am J Pathol 1998;152:1071-9.

52. Chen B, He L, Savell VH, Jenkins JJ, Parham DM. Inhibition of the interferon-gamma/signal transducers and activators of transcription (STAT) pathway by hypermethylation at a STAT-binding site in the p21WAF1 promoter region. Cancer Res 2000;60:3290-8.

53. Jung WH, Jung SH, Yoo CJ, Kim YJ, Park C, Kim BS. Flow cytometric analysis of DNA ploidy in childhood rhabdomyosarcoma. Yonsei Med J 1994;35:34-42.

54. Niggli FK, Powell JE, Parkes SE, Ward K, Raafat F, Mann JR, et al. DNA ploidy and proliferative activity (S-phase) in childhood soft- tissue sarcomas: their value as prognostic indicators. Br J Cancer 1994;69:1106-10.

55. Bridge JA, Liu J, Weibolt V, Baker KS, Perry D, Kruger R, et al. Novel genomic imbalances in embryonal rhabdomyosar- coma revealed by comparative genomic hybridization and fluorescence in situ hybridization: an intergroup rhabdomyosarcoma study. Genes Chromosomes Cancer 2000;27: 337-44.

56. Lee W, Han K, Harris CP, Meisner LF. Detection of aneuploidy and possible deletion in paraffin- embedded rhabdomyosarcoma cells with FISH. Cancer Genet Cytogenet 1993; 68:99-103.

57. Smith SS. Stalling of DNA methyltransferase in chromosome stability and chromosome remodelling. Int J Mol Med 1998; 1:147-56.

58. Vilain A, Vogt N, Dutrillaux B, Malfoy B. DNA methylation and chromosome instability in breast cancer cell lines. FEBS Lett 1999;460:231-4.

59. $\mathrm{Xu}$ GL, Bestor TH, Bourc'his D, Hsieh CL, Tommerup N, Bugge $\mathrm{M}$, et al. Chromosome instability and immunodeficiency syndrome caused by mutations in a DNA methyltransferase gene. Nature 1999;402:187-91.

60. Dias P, Chen B, Dilday B, Palmer H, Hosoi H, Singh S, et al. Strong immunostaining for myogenin in rhabdomyosarcoma is significantly associated with tumors of the alveolar subclass. Am J Pathol 2000;156:399-408.

61. Pinto A, Tallini G, Novak RW, Bowen T, Parham DM. Undifferentiated rhabdomyosarcoma with lymphoid phenotype expression. Med Pediatr Oncol 1997;28:165-70.

62. Boue D, Parham D, Webber B, Maurer H, Qualman S. Clinicopathologic study of pediatric ectomesenchymoma from IRS III and IV [Abstract]. Mod Pathol 1997;10:2P.

63. Etcubanas E, Peiper S, Stass S, Green A. Rhabdomyosarcoma, presenting as disseminated malignancy from an unknown primary site: a retrospective study of ten pediatric cases. Med Pediatr Oncol 1989;17:39-44.

64. Coffin CM, Rulon J, Smith L, Bruggers C, White FV. Pathologic features of rhabdomyosarcoma before and after treatment: a clinicopathologic and immunohistochemical analysis. Mod Pathol 1997;10:1175-87.

65. Andrassy RJ, Wiener ES, Raney RB, Hays DM, Arndt CA, Lobe TE, et al. Progress in the surgical management of vaginal rhabdomyosarcoma: a 25-year review from the Intergroup Rhabdomyosarcoma Study Group. J Pediatr Surg 1999;34: 731-4. 